\title{
Exosomes Derived from Cyclic Mechanical Stretch- exposed Bone Marrow Mesenchymal Stem Cells Inhibit RANKL-induced Osteoclastogenesis through the NF-kB Signaling Pathway
}

Fei Xiao

Shanghai Jiaotong University School of Medicine Xinhua Hospital

Bin Zuo

Shanghai Jiaotong University School of Medicine Xinhua Hospital

Chuandong Wang

Shanghai Jiaotong University School of Medicine Xinhua Hospital

\section{Yang Li}

Shanghai Jiaotong University School of Medicine Xinhua Hospital Jianping Peng

Shanghai Jiaotong University School of Medicine Xinhua Hospital

Chao Shen

Shanghai Jiaotong University School of Medicine Xinhua Hospital

\section{Yiming Cui}

Shanghai Jiaotong University School of Medicine Xinhua Hospital

\section{Zhu Junfeng}

Shanghai Jiaotong University School of Medicine Xinhua Hospital

Xiaodong Chen ( $\nabla$ chenxiaodong@xinhuamed.com.cn)

Shanghai Jiaotong University School of Medicine Xinhua Hospital

\section{Research}

Keywords: Exosome, Mechanical loading, Bone marrow mesenchymal stem cell, Osteoclast, Osteoporosis

Posted Date: December 1st, 2020

DOl: https://doi.org/10.21203/rs.3.rs-115772/v1

License: (c) (1) This work is licensed under a Creative Commons Attribution 4.0 International License.

Read Full License 


\section{Abstract}

Background: Skeletal unloading usually induces severe disuse osteoporosis (DOP), which often occurs in patients subjected to prolonged immobility or in spaceflight astronauts. Increasing evidence suggests that exosomes are important mediators in maintaining the balance between bone formation and bone resorption. We hypothesized that exosomes play a pivotal role in the maintenance of bone homeostasis through intercellular communication between bone marrow mesenchymal stem cells (BMSCs) and osteoclasts under mechanical loading.

Methods: Cells were divided into cyclic mechanical stretch (CMS)-treated BMSCs and normal staticcultured BMSCs, and exosomes were extracted by ultracentrifugation. After incubation with CMS-treated BMSC-derived exosomes (CMS_Exos) or static-cultured BMSC-derived exosomes (static_Exos), the apoptosis rates of bone marrow macrophages (BMMs) were determined by flow cytometry, and cell viability was detected with a CCK-8 assay. Osteoclast differentiation was determined with an in vitro osteoclastogenesis assay. Signaling pathway activation was evaluated by Western blotting and immunofluorescence staining. Hindlimb unloading (HU)-induced DOP mouse models were prepared to evaluate the function of exosomes in DOP.

Results: Both CMS_Exos and static_Exos could be internalized by BMMs, and CMS_Exos did not affect BMM viability or increase apoptosis. CMS_Exos effectively suppressed receptor activator of nuclear factor-KB ligand (RANKL)-mediated osteoclast differentiation and F-actin ring formation. Further molecular investigation demonstrated that CMS_Exos impaired osteoclast differentiation via inhibition of the RANKL-induced NF-KB signaling pathway. Both CMS_Exos and static_Exos partly rescued the osteoporosis caused by mechanical unloading; however, the CMS_Exo group showed more obvious rescue. CMS_Exo treatment significantly decreased the number of tartrate-resistant acid phosphatase (TRAP)-positive osteoclasts.

Conclusions: Exosomes derived from CMS-treated BMSCs strongly inhibited osteoclast differentiation by attenuating the NF-KB signaling pathway in vitro and rescued osteoporosis caused by mechanical unloading in an $\mathrm{HU}$ mouse model in vivo.

\section{Background}

Bone is a rigid but dynamic tissue that undergoes modeling and remodeling throughout life. Mechanical loading has been previously reported to play a crucial role in modulating bone remodeling and homeostasis [1-3]. Under normal weight-bearing conditions, bone remodeling is coordinately regulated by osteoclastic bone resorption and osteoblastic bone formation. Reduced mechanical stimulation of bone disrupts this delicate equilibrium and leads to significant bone loss, as evidenced by disuse osteoporosis (DOP), which is a critical issue for long-term bed rest patients and astronauts [4-6].

Osteoblasts and osteoclasts, including their precursor cells, are sensitive to mechanical stimuli and can transform mechanical signals into biological responses [1, 2, 4-9]. Proper mechanical stimulation 
promotes the osteogenic differentiation of bone marrow mesenchymal stem cells (BMSCs) through epigenetic regulation via HDAC1 and Dnmt3b [6, 9]. Our previous study demonstrated that mechanical loading positively regulates bone formation in vivo and that cyclic mechanical stretch (CMS) regulates osteoblast differentiation in vitro via mechanosensitive miRNA-103a directly targeting Runx2 [1]. However, RANKL-induced osteoclast differentiation is directly suppressed during the period of mechanical loading but enhanced in the absence of mechanical stress $[5,7,8]$. Previous studies have focused on the mechanical responses during the differentiation of osteoblasts and osteoclasts, but the intercellular communication between these cells under mechanical loading has not been fully elucidated.

Intercellular communication between osteoblasts and osteoclasts is important for the establishment and maintenance of bone remodeling [10-12]. In addition, BMSCs not only act as progenitors of osteoblasts but also support osteoclast differentiation and participate in communication related to bone remodeling [13]. Recently, exosomes, extracellular membrane vesicles with a diameter of $\sim 30-160 \mathrm{~nm}$, have been demonstrated to traffic RNA, DNA, and proteins between cells and serve as mediators of intercellular communication $[14,15]$. Studies have revealed the involvement of a number of bone-derived exosomes in the regulation of bone homeostasis $[13,14,16-19]$. For example, exosomes derived from UAMS-32P, an osteoblastic cell line, facilitate osteoclast formation [20]. In contrast, osteoclast-derived miR-214-3pcontaining exosomes can be transferred to osteoblasts to inhibit osteoblast activity and bone formation $[10,21]$. To date, a large number of studies have confirmed that osteoclast-derived factors and exosomepackaged microRNAs can either inhibit or enhance osteoblast differentiation through juxtacrine or paracrine mechanisms independent of their resorptive activity [17]. However, research focusing on BMSCderived exosomes influencing osteoclast differentiation is rare [18].

We hypothesized that exosomes play a pivotal role in the maintenance of bone homeostasis through mediating intercellular communication between BMSCs and osteoclasts under mechanical loading. In this study, we demonstrated, for the first time, that exosomes derived from CMS-treated BMSCs (CMS_Exos) strongly inhibited osteoclast differentiation by attenuating the NF-kB signaling pathway. Furthermore, these exosomes effectively rescued osteoporosis caused by mechanical unloading in a hindlimb unloading $(\mathrm{HU})$ mouse model in vivo. These results not only demonstrate the role of exosomes in communication between BMSCs and osteoclasts under mechanical loading but also reveal a potential therapeutic approach for DOP.

\section{Materials And Methods}

\section{Cell culture}

BMSCs were obtained from C57BL/6J mice and cultured as previously described [6]. Briefly, BMSCs were flushed out of the femurs and tibias with a-minimum essential medium (a-MEM) and cultured in growth medium (a-MEM supplemented with $10 \%$ fetal bovine serum [FBS], $100 \mathrm{U} / \mathrm{mL}$ penicillin $\mathrm{G}$, and $100 \mu \mathrm{g} / \mathrm{mL}$ streptomycin [all from HyClone, Logan, UT, USA]) at $37{ }^{\circ} \mathrm{C}$ in the presence of $5 \% \mathrm{CO}_{2}$ after lysis of red blood cells. Nonadherent cells were removed by replacing the growth medium after 3 days. The attached 
BMSCs were not used beyond passage 5. Bone marrow macrophages (BMMs) were prepared as previously described [22]. Briefly, cells extracted from the femurs and tibias of mice were allowed to proliferate in growth medium containing $30 \mathrm{ng} / \mathrm{mL}$ M-CSF (R\&D Systems, Minneapolis, MN, USA). The cells were washed during a medium change to deplete residual stromal cells. At $80 \%-90 \%$ confluence, the cells were washed with phosphate-buffered saline (PBS) three times and then trypsinized to harvest BMMs.

\section{CMS application}

BMSCs were seeded on six-well flexible silicone rubber BioFlex plates coated with collagen type I (Flexcell International Corporation, Hillsborough, NC, USA). The cells were cultured for 24 to $36 \mathrm{~h}$ to reach $60 \%$ confluency, at which time the growth medium was replaced. CMS at a $0.5-\mathrm{Hz}$ sinusoidal curve at $8 \%$ elongation was applied using an FX-5000T Flexercell Tension Plus unit (Flexcell International Corporation) as described in our previous study [1]. The cells were incubated in a humidified atmosphere at $37^{\circ} \mathrm{C}$ and $5 \% \mathrm{CO}_{2}$ during stretching. The conditioned medium for exosome collection was supplemented with FBS previously depleted of exosomes by ultracentrifugation at 120,000 g for $90 \mathrm{~min}$. The culture supernatants were harvested immediately after CMS stimulation for $48 \mathrm{~h}$. Control cells were cultured on the same plates in the same incubator but were not subjected to stretching.

\section{Exosome extraction and identification}

Exosome extraction was performed as previously described [10,21]. First, culture supernatant containing exosomes was harvested by centrifugation at $300 \mathrm{~g}$ for $10 \mathrm{~min}$ at $4{ }^{\circ} \mathrm{C}$ to remove floating cells, followed by additional rounds of centrifugation at $2,000 \mathrm{~g}$ for $20 \mathrm{~min}$ and $10,000 \mathrm{~g}$ for $5 \mathrm{~min}$ at $4{ }^{\circ} \mathrm{C}$ and passage through a $0.22-\mu \mathrm{m}$ membrane filter to remove particles larger than $220 \mathrm{~nm}$. At each step, the supernatant was transferred to new tubes, and the pellets were immediately rinsed with PBS. Exosomes were pelleted via ultracentrifugation at $120,000 \mathrm{~g}$ for $70 \mathrm{~min}$ at $4{ }^{\circ} \mathrm{C}$, washed in PBS and ultracentrifuged again, and the pellets were resuspended in PBS. The isolated exosomes were used immediately or stored at $-80^{\circ} \mathrm{C}$. The protein concentration in exosomes was determined with a BCA protein assay kit (Thermo Scientific, Product \#23225). Exosome morphology was detected by transmission electron microscopy (TEM). The size distribution and intensity of exosomes were examined using NanoSight (NanoSight NS300, Malvern, UK). Specific exosomal biomarkers, including CD63 and TSG101, were detected by Western blot analysis.

\section{Exosome labeling and uptake}

The fluorescent dye 3,3'-dioctadecyloxacarbocyanine perchlorate (DiO) (Beyotime, Shanghai, China) was used to label exosomes and the cell membrane. CMS_Exos and exosomes derived from control BMSCs (static_Exos) were incubated with the fluorescent dye DiO $(5 \mathrm{mM})$ for $15 \mathrm{~min}$ at $37^{\circ} \mathrm{C}$, followed by two rounds of ultracentrifugation at 120,000 $\mathrm{g}$ for $90 \mathrm{~min}$ to remove any free dye. The labeled exosomes were incubated with BMMs for $4 \mathrm{~h}$. After that, the cells were fixed and incubated with DAPI (Beyotime, Shanghai, China) to stain cell nuclei. The uptake of labeled exosomes by BMMs was observed with an LSM5 confocal microscope (Zeiss, Germany).

\section{Measurement of cell viability and apoptosis}


According to the manufacturer's instructions for a Cell Counting Kit-8 (CCK-8) kit, the antiproliferative effect of exosomes on BMMs was assessed. Briefly, BMMs were seeded and then cultured for $48 \mathrm{~h}$ with different doses of exosomes. Cell viability was examined using CCK-8 (Dojindo, Japan). Optical density was measured at $450 \mathrm{~nm}$ (630 nm as reference) with an ELX800 absorbance microplate reader (Bio-Tek, Winooski, VT, United States). Detection of cell apoptosis rates was performed by flow cytometry using the FITC Annexin V Apoptosis Detection Kit I (BD Biosciences, USA). BMMs were exposed to exosomes for $48 \mathrm{~h}$ in the presence or absence of RANKL. The results were analyzed with a FACStar (BD Biosciences). Nuclei were stained with DAPI in PBS at $37^{\circ} \mathrm{C}$ for $10 \mathrm{~min}$ in the dark. The cell nuclei were observed and imaged with confocal microscopy.

\section{In vitro osteoclastogenesis assay}

BMMs were differentiated into osteoclasts over 5 days by incubation in complete culture medium containing RANKL (50 ng/mL) and M-CSF (30 ng/mL), as previously described [22]. To determine the influence of exosomes on osteoclast differentiation, exosomes were included in the culture medium at a range of concentrations $(0,510$ or $25 \mu \mathrm{g} / \mathrm{mL})$. Every other day during the incubation, the culture medium was replenished with fresh medium containing RANKL, M-CSF, and the indicated concentration of exosomes. To investigate all stages of exosome addition-dependent effects on osteoclastogenesis, BMMs were cultured with exosomes $(25 \mu \mathrm{g} / \mathrm{mL})$ added at different stages of the 5-day culture period: days $0-2$ (early stage) and days 3-5 (late stage). After 5 days, cells were fixed in paraformaldehyde and stained for tartrate-resistant acid phosphatase (TRAP) activity. The number of mature osteoclasts (TRAP ${ }^{+}$ cells containing more than three nuclei) was calculated.

\section{F-actin ring immunofluorescence}

For F-actin ring immunofluorescence staining, exosome-pretreated osteoclasts were fixed in $4 \%$ paraformaldehyde for 15 min, permeabilized in $0.1 \%$ Triton X-100 in PBS for 5 min, and then incubated with rhodamine-conjugated phalloidin for $30 \mathrm{~min}$ at room temperature. The cells were then incubated with DAPI for visualization of nuclei. The actin ring distribution was visualized using a confocal microscope.

\section{Confocal microscopy for NF-KB localization}

RAW264.7 cells were seeded in 6-well plates containing sterile cover slips. After incubation with culture medium at $37^{\circ} \mathrm{C}$ for $24 \mathrm{~h}$, the cells were pretreated with exosomes for $4 \mathrm{~h}$, followed by stimulation with RANKL ( $50 \mathrm{ng} / \mathrm{mL}$ ) for $20 \mathrm{~min}$. After the incubation, the cells were washed twice with PBS, fixed in $4 \%$ paraformaldehyde for $20 \mathrm{~min}$ and then permeabilized with $0.1 \%$ Triton X-100 for 30 min at room temperature. After blocking with $5 \% \mathrm{BSA}$ for $1 \mathrm{~h}$ at room temperature, the cells were incubated with an anti-p65 subunit antibody (Cell Signaling Technology, Danvers, USA) diluted 1:100 in PBS at $4{ }^{\circ} \mathrm{C}$ overnight. Nuclei were stained with DAPI. The nuclear translocation of p65 was imaged using confocal microscopy.

\section{Western blot analysis}


Western blot analysis was performed as previously described [22]. RAW264.7 cells were seeded in 6-well plates at a density of $6 \times 10^{5}$ cells per well. After growing to confluence, the cells were pretreated with or without exosomes for $4 \mathrm{~h}$. The cells were then stimulated with $50 \mathrm{ng} / \mathrm{mL}$ RANKL for $10 \mathrm{~min}$. The cells were lysed with radioimmunoprecipitation assay lysis buffer (Thermo Fisher Scientific, Waltham, MA, USA), and the protein concentration was determined with a BCA protein assay. Lysate proteins $(30 \mu \mathrm{g})$ were separated by $10 \%$ SDS-PAGE and transferred to polyvinylidene difluoride membranes. The membranes were blocked with $5 \%$ skim milk in TBST for $1 \mathrm{~h}$ and incubated with primary antibodies overnight at $4{ }^{\circ} \mathrm{C}$. An HRP-conjugated secondary antibody was used at a 1:5,000 dilution. Immunoblots were visualized with an enhanced chemiluminescence detection system (Millipore, Billerica, MA, USA). Immunoreactive bands were quantified on scanned films in triplicate by normalizing the band intensities to the intensity of the GAPDH band with the help of Alpha Image software. All primary antibodies and secondary antibodies used in this study were purchased from Cell Signaling Technology.

\section{Hindlimb-unloading mice}

Animal studies were performed as previously described [1]. Six-month-old male C57BL/6J mice were purchased from Shanghai SLAC Laboratory Animal Co., Ltd. and were individually caged under standard conditions (12-h light/12-h dark cycle, $21^{\circ} \mathrm{C}$ controlled temperature). Animals were suspended from the hindlimbs for a period of 28 days. The suspension height was adjusted to prevent the hindlimbs from touching any supporting surface while maintaining a suspension angle of approximately 30 degrees. This allowed the forelimbs to have contact with the grid floor and allowed the animals to move around the cage for free access to food and water. Mice in the intervention groups received tail vein injections of Static_Exos or CMS_Exos ( $5 \mathrm{mg} / \mathrm{kg}$ body weight, $0.1 \mathrm{ml}$ per injection) twice a week for 4 weeks. In the weight-bearing (WB) group or HU group, the mice received an equal volume of PBS at the same time points. Four weeks later, the bilateral femurs and tibias were dissected and processed for micro-computed tomography $(\mu \mathrm{CT})$ examination and bone histomorphometric analysis.

\section{$\mu \mathrm{CT}$ analysis of femurs}

Right femurs were fixed in $4 \%$ paraformaldehyde for $24 \mathrm{~h}$ and stored in $70 \%$ ethanol at $4{ }^{\circ} \mathrm{C}$. The samples were scanned by high-resolution $\mu$ CT (SCANCO $\mu$ CT 100, Brüttisellen, Switzerland). Each femur was imaged with the following instrument settings: 70 kV, $200 \mu \mathrm{A}, 0.5-\mathrm{mm}$ Al filter, 300-ms exposure, and 10$\mu \mathrm{m}$ pixel size. After scanning, the data were reconstructed with SCANCO $\mu 100$ Evaluation V6.5-3 with a constant threshold value. The region of interest (ROI) of trabecular bone was $0.8 \mathrm{~mm}$ in height and $0.3 \mathrm{~mm}$ below the growth plate, and the ROI of cortical bone was $1 \mathrm{~mm}$ in height and $2 \mathrm{~mm}$ below the growth plate. The ROI of bone within this volume was manually defined, and the following bone parameters were included: Tb.Th, BMD, bone volume percentage (BV/TV), Tb.N, Tb.Sp and Ct.Th.

\section{Histological and histomorphometric analysis}

Left femurs were fixed in $4 \%$ paraformaldehyde for $24 \mathrm{~h}$, and then $12.5 \%$ EDTA was used for decalcification for approximately 3 weeks. The tissues were embedded in paraffin, and histological sections (5- $\mu \mathrm{m}$ thick) were made. Subsequently, the sections underwent hematoxylin and eosin (H\&E) and 
TRAP staining with methyl green counterstaining. A high-quality microscope was used to examine and image the specimens. The number of $\mathrm{TRAP}^{+}$multinucleated osteoclasts (N.Oc/BS, $1 / \mathrm{mm}$ ) and the percentage of osteoclast surface per bone surface (OcS/BS, \%) were assessed for each sample.

\section{Statistical analysis}

SPSS 22.0 software (SPSS Inc., United States) was included for statistical analysis. All values are presented as the mean \pm standard deviation (SD) of three independent replicates. Student's t-test was performed to compare differences. $* p<0.05$ and $* * p<0.01$ indicate significant differences.

\section{Results}

\section{Characterization of exosomes and incorporation of exosomes into BMMs}

Exosomes were successfully isolated from BMSC culture medium and identified (Fig. 1). The exosomes were observed using TEM, revealing a typical diameter of $\sim 100-150 \mathrm{~nm}$ with a rounded morphology (Fig. 1a). Particle size and concentration were measured by NanoSight (Fig. 1b). The diameters of Static_Exos were distributed at $60-200 \mathrm{~nm}$ with a size peak of $98 \mathrm{~nm}$, and the diameters of CMS_Exos were distributed at 40-260 $\mathrm{nm}$ with a size peak of $105 \mathrm{~nm}$. Western blot analysis results confirmed presence of expected proteins, such as CD63 and TSG101, in the exosomes and the absence of the nuclear proteins LaminA/C and the endoplasmic reticulum protein Calnexin (Fig. 1c).

To assess whether exosomes derived from BMSCs can be internalized by BMMs, exosomes were labeled with DiO (green). The labeled exosomes were incubated with BMMs for $4 \mathrm{~h}$ at $37^{\circ} \mathrm{C}$. The cells were then observed by confocal microscopy, which revealed the incorporation of exosomes into BMMs (Fig. 1d).

\section{CMS_Exos inhibit RANKL-induced osteoclastogenesis and actin ring formation}

To examine the effects of exosomes derived from BMSCs on RANKL-induced osteoclastogenesis in vitro, a cell viability test was performed first. Concentrations of up to $50 \mu \mathrm{g} / \mathrm{mL}$ Static_Exos or CMS_Exos had no cytotoxic effects on BMMs, which were treated with Static_Exos or CMS_Exos for $48 \mathrm{~h}$ (Fig. 3e). BMMs were treated with M-CSF and RANKL in the absence or presence of different concentrations of exosomes for 5 days. Numerous TRAP + multinucleated osteoclasts were formed under stimulation with M-CSF and RANKL, whereas osteoclast formation was inhibited by CMS_Exo treatment in a dose-dependent manner (Fig. 3a, b). However, Static_Exo treatment showed no significant inhibitory effect on osteoclast formation (Fig. 3a, b). To examine the stage at which CMS_Exos inhibit osteoclastogenesis, $25 \mu \mathrm{g} / \mathrm{mL}$ exosomes was added to the culture medium at 0 or 3 days during osteoclast differentiation. CMS_Exo treatment during the early stage (days $0-2$ ) significantly suppressed osteoclastogenesis (Fig. 3c, d). Furthermore, exposure of osteoclastic precursor cells to CMS_Exos during the later stage (days 3-5) also 
affected osteoclast formation, but the effect was weaker than that of treatment during the early stage (Fig. 3c, d). Thus, CMS_Exos inhibited osteoclast differentiation during both the early and late stages.

F-actin rings are an important constituent of osteoclasts and a prerequisite for efficient bone resorption. Thus, we further assessed the effect of CMS_Exos on F-actin ring formation. Clear actin ring structures were observed in the vehicle treatment group and Static_Exo group (Fig. 3e). However, the actin ring structure was almost completely disrupted when BMMs were incubated with $25 \mu \mathrm{g} / \mathrm{mL}$ CMS_Exos (Fig. 3e, f).

To exclude the possibility that exosomes induced BMM apoptosis during differentiation, BMMs were treated with Static_Exos or CMS_Exos in the absence or presence of RANKL for $48 \mathrm{~h}$. The number of Annexin- $V+$ cells was evaluated by flow cytometry. BMMs treated with CMS_Exos as a concentration of $25 \mu \mathrm{g} / \mathrm{mL}$ showed suppressed osteoclast formation, but the number of Annexin-V + BMMs was not affected by CMS_Exo treatment at $48 \mathrm{~h}$ compared with control treatment (Fig. 2a-c). Furthermore, BMMs stained with DAPI exhibited normal intact nuclei after CMS_Exo treatment (Fig. 2d, e), thereby confirming that the inhibitory effects of CMS_Exos on osteoclastogenesis were not caused by the induction of BMM apoptosis.

\section{CMS_Exos inhibit RANKL-induced activation of the NF-KB signaling pathway}

Activation of the NF-KB signaling pathway plays a crucial role in osteoclastogenesis. To further explore the underlying mechanisms through which CMS_Exos regulate osteoclast formation, RANKL-induced signaling pathways were investigated. To observe the impact of exosomes on NF-KB signaling directly, immunofluorescence staining was performed to analyze the nuclear translocation of NF-kB/p65 (Fig. 4a). Negative control cells were not exposed to RANKL or exosomes. These results clearly showed that compared with the negative control cells, RANKL-stimulated Raw264.7 cells showed obvious translocation of p65 (red) from the cytoplasm to the nucleus (blue) and that this translocation could be prevented by CMS_Exos. However, Static_Exos did not affect p65 translocation. Furthermore, Western blotting revealed changes in NF-KB pathway protein levels (Fig. 4b, c). NF-KB pathway-related proteins, including inhibitor of NF-KB (IKB)a, IKB kinase (IKK), and p65, were phosphorylated or activated under RANKL stimulation. ІкBa degradation allows activated NF-KB/p65 to translocate into the nucleus and regulate the expression of certain genes. However, this process was inhibited effectively by CMS_Exos but not by Static_Exos (Fig. 4c). Additionally, the phosphorylation of IKKa/ $\beta$ was suppressed by CMS_Exos but not by Static_Exos in the context of RANKL stimulation (Fig. 4c). Therefore, the inhibitory effects of CMS_Exos on NF-KB signaling may play a pivotal role in the inhibition of RANKL-stimulated osteoclastogenesis.

\section{CMS_Exos prevented HU-induced bone loss}


To evaluate the role of exosomes in DOP, we established an HU-induced bone loss mouse model. There were no fatalities during $\mathrm{HU}$ treatment or exosome administration. $\mu \mathrm{CT}$ confirmed that there was significant loss of femur trabecular and cortical bone in $\mathrm{HU}$ mice, which was indicated by decreased BMD, BV/TV, Tb.Th, Tb.N and Ct.Th values as well as an increased Tb.Sp value for HU mice compared to WB mice (Fig. 5a, b). However, the extent of trabecular bone loss was significantly suppressed by treatment with CMS_Exos. The relative bone loss in mice treated with CMS_Exos compared with that in HU mice treated with the vehicle was markedly less than that in mice treated with Static_Exos (Fig. 5a, b). Cortical bone loss was ameliorated by treatment with CMS_Exos but not Static_Exos, indicating that CMS_Exos have a more comprehensive antiosteoporosis function. In agreement with the $\mu \mathrm{CT}$ analysis results, histological H\&E-stained sections revealed that the trabeculae in regions proximal and distal to the growth plate were thin in the HU mouse group (Fig. 5c). In contrast, HU mice treated with CMS_Exos exhibited a dramatic increase in bone density and marked increases in trabecular density and thickness compared with HU mice treated with the vehicle (Fig. 5c).

To further explore whether osteoclasts are involved in the inhibitory effects of exosomes on HU-induced bone loss, we performed TRAP staining on femur bone sections. Accordingly, TRAP staining revealed a significant decrease in the numbers of TRAP + multinucleated cells at the growth plates and trabecular surface of CMS_Exo-treated mice compared with vehicle-treated HU mice (Fig. 5c). Moreover, histomorphometric analysis of Oc.S/BS and the number of osteoclasts confirmed that CMS_Exo treatment attenuated HU-induced bone loss and reduced osteoclast numbers (Fig. 5d). Although Static_Exo treatment rescued trabecular bone loss in mice to a certain extent, it did not show significant inhibition of osteoclasts in vivo, manifesting as no significant reductions in the number or area of osteoclasts on the bone surface (Fig. 5c, d). Collectively, these results indicated that CMS_Exos effectively prevented HU-induced bone loss in vivo.

\section{Discussion}

BMSCs have been proposed to be promising candidates for the treatment of various diseases, such as muscle, bone and cartilage diseases; blood-related diseases; immune system diseases; nervous system diseases; and injuries [23]. Recently, it was identified that BMSCs exert their therapeutic activity through a paracrine effect mediated by the release of small extracellular vesicles (EVs) [16]. Exogenous BMSC-EVs can be taken up and promote the osteogenic differentiation of endogenous BMSCs [24]. Moreover, BMSCEVs can be endocytosed by osteoblasts and release their cargo into target cells to positively regulate osteogenic genes and osteoblastic differentiation [25]. However, studies focusing on BMSC-derived exosomes influencing osteoclast differentiation are rare [18]. In this study, we investigated the effects of exosomes derived from static or mechanically stimulated BMSCs on osteoclast differentiation. The results indicated that CMS_Exos effectively inhibited RANKL-induced osteoclastogenesis by attenuating the NF-KB signaling pathway in vitro. Moreover, CMS_Exos rescued osteoporosis in a mechanical unloading mouse model in vivo. 
Mechanical stimulation has been reported to be an important regulator in diverse biological and pathological processes, including affecting the secretion of EVs and their cargoes [1, 4, 26, 27]. Recently, studies have shown that both cyclic stretching and continuous stretching regulate the expression of specific microRNAs (miRNAs) in EVs in lung epithelial cells and regulate lung development [26]. Exosomes from cyclic stretch-treated periodontal ligament cells contribute to the maintenance of periodontal immune/inflammatory homeostasis by inhibiting IL-1 $\beta$ production and pyroptosis in macrophages [27]. In the present study, we characterized exosomes secreted by static culture- or mechanical stretch-treated BMSCs (Fig. 1a-c). The uptake of these exosomes by BMMs was observed by confocal microscopy, confirming a direct interaction between BMSC-derived exosomes and BMMs (Fig. 1d). Neither indicated concentration CMS_Exo treatment nor indicated concentration Static_Exo treatment affected the apoptosis of BMMs in the presence or absence of RANKL (Fig. 2). Interestingly, CMS_Exo treatment produced sustained osteoclast differentiation inhibition, while Static_Exo treatment did not (Fig. 3), indicating that mechanical stimulation altered the cargo profile of BMSC-derived exosomes. To our knowledge, this is the first report about the effects of BMSC-derived exosomes on osteoclast differentiation.

During osteoclast differentiation, RANKL binding to RANK induces the activation of several vital signaling pathways. As an essential signaling pathway in most cells, the strong activation of NF-KB by RANKL plays a crucial role in osteoclast differentiation, resorption function and survival [28]. Thus, the RANKLinduced changes in the levels of proteins involved in NF-KB signaling were investigated in this study. When BMMs are stimulated with RANKL, IKB is phosphorylated by the IKB kinase complex (IKKa, IKK $\beta$, and NEMO), ubiquitinated, and degraded by the ubiquitin-proteasome system. Then, free NF-KB proteins (such as p65) translocate into the nucleus and recognize DNA target sites to trigger the expression of osteoclastogenesis-related genes [29]. However, it was found that the degradation of IKBa and phosphorylation of p65, IKKa/ $\beta$ and IKBa were effectively blocked by CMS_Exo exposure but not by Static_Exo exposure (Fig. 4b, c). Moreover, immunofluorescence analysis showed that CMS_Exos disturbed p65 nuclear translocation, thus preventing the activation of downstream factors (Fig. 4a). In short, CMS_Exos attenuated osteoclast formation and function via inhibition of the NF-kB signaling pathway.

Increasing evidence has shown the therapeutic potential of exosomes in treating various bone diseases $[30,31]$. The bone mineral density of bovine colostrum exosome-fed glucocorticoid-induced osteoporosis mice was significantly improved compared with that of control mice without exosome treatment [32]. In a femoral fracture model, mice treated with exosomes from human umbilical cord MSCs (HUCMSCs) cultured under hypoxic conditions showed a significantly larger callus volume and greater vascularity in the fracture site than those treated with exosomes from HUCMSCs cultured under normoxic conditions or with PBS [33]. Recently, exosomes derived from HUCMSCs were shown to have therapeutic effects on a rat DOP model [34]. However, this study only focused on the apoptosis of BMSCs, and did not investigate the biological behavior of osteoclasts and the intercellular communication between BMSCs and osteoclasts. Mechanical signals play critical roles in bone growth and homeostasis [35, 36], and we hypothesized that exosomes play a pivotal role in the maintenance of bone homeostasis through 
mediating intercellular communication between BMSCs and osteoclasts under mechanical loading. Indeed, Static_Exos decreased bone loss to a certain extent, as shown in Fig. 5a and b. The bone loss in mice treated with CMS_Exos compared with that in HU mice treated with the vehicle was markedly less than that in mice treated with Static_Exos. Moreover, cortical bone loss was ameliorated by treatment with CMS_Exos but not Static_Exos. This may be because mechanical stimulation changes the components of exosome cargoes, which makes special exosomes exhibit superior anti-DOP activity. It is not clear which component is responsible for this effect, and further investigations identifying the specific content and exploring the underlying molecular mechanisms are needed to optimize the efficacy of CMS_Exos for DOP therapy.

\section{Conclusions}

In summary, our study provides new insights into intercellular communication between osteoblasts and osteoclasts under mechanical loading. Exosomes derived from CMS-treated BMSCs suppressed osteoclastogenesis by attenuating NF-KB signaling pathway activity in vitro and ameliorated bone loss caused by mechanical unloading in an HU mouse model. In-depth studies on CMS_Exo components, such as proteins and miRNAs, are required to understand how CMS_Exos function at the molecular level.

\section{Abbreviations}

DOP: Disuse osteoporosis; BMSCs: Bone marrow mesenchymal stem cells; CMS: Cyclic mechanical stretch; BMMs: Bone marrow macrophages; HU: Hindlimb unloading; RANKL: Receptor activator of nuclear factor-KB ligand; TRAP: Tartrate-resistant acid phosphatas; a-MEM: a-minimum essential medium; TEM: Transmission electron microscopy; DiO: 3,3'-dioctadecyloxacarbocyanine perchlorate; CCK-8: Cell Counting Kit-8; PBS: Phosphate-buffered saline; BV/TV: Bone volume to total tissue volume ratio; Tb.Th: Trabecular thickness, Tb.N :Trabecular number; Tb.Sp: Trabecular separation; Ct.Th: Cortical bone thickness; BMD: Bone mineral density; N.Oc/BS: Osteoclast surface to bone surface ratio.

\section{Declarations}

\section{Acknowledgement}

Not applicable.

\section{Funding}

This study was sponsored by Shanghai Sailing Program (20YF1429100), Shanghai Natural Science Fund (19ZR1433100), Interdisciplinary of Medicine and Engineering Foundation of Shanghai Jiao Tong University (YG2019ZDA22), Biomedical Technology Support Program of Shanghai (20S31900200).

\section{Availability of data and materials}


All data generated or analyzed during this study are included in this article.

\section{Authors' contributions}

FX and BZ contributed to the study and data collecting of this manuscript. JFZ and XDC contributed to the conception and design, financial and administrative support, and manuscript reviewing and revising. CDW, YL and JPP contributed to the data collection and analysis. CS and YMC contributed to the data interpretation and manuscript reviewing and revising. FX, and BZ contributed equally to this work. All authors read and approved the final manuscript.

\section{Ethics approval and consent to participate}

All animal studies were conducted in accordance with the ethical standards set by the Declaration of Helsinki and approved by the Institutional Ethics Committee of Xinhua Hospital affiliated to Shanghai Jiao Tong University School of Medicine. It met the NIH guidelines for the care and use of laboratory animals.

\section{Consent for publication}

Not applicable.

\section{Competing interests}

The authors declare that they have no competing interests.

\section{References}

1. Zuo B, Zhu JF, Li J, Wang CD, Zhao XY, Cai GQ, Li Z, Peng JP, Wang P, Shen C, Huang Y, Xu JK, Zhang $X \mathrm{~L}$, Chen XD. microRNA-103a functions as a mechanosensitive microRNA to inhibit bone formation through targeting Runx2. J Bone Miner Res. 2015;30:330-45.

2. Hsieh $\mathrm{YF}$, Turner $\mathrm{CH}$. Effects of loading frequency on mechanically induced bone formation. J Bone Miner Res. 2001;16:918-24.

3. Yan YY, Wang LP, Ge LH, Pathak JL. Osteocyte-Mediated Translation of Mechanical Stimuli to Cellular Signaling and Its Role in Bone and Non-bone-Related Clinical Complications. Curr Osteoporos Rep. 2020;18:67-80.

4. Li J, Wan Z, Liu H, Li H, Liu L, Li R, Guo Y, Chen W, Zhang X, Zhang X. Osteoblasts subjected to mechanical strain inhibit osteoclastic differentiation and bone resorption in a co-culture system. Ann Biomed Eng. 2013;41:2056-66.

5. Nabavi N, Khandani A, Camirand A, Harrison RE. Effects of microgravity on osteoclast bone resorption and osteoblast cytoskeletal organization and adhesion. Bone. 2011;49:965-74.

6. Wang C, Shan S, Wang C, Wang J, Li J, Hu G, Dai K, Li Q, Zhang X. Mechanical stimulation promote the osteogenic differentiation of bone marrow stromal cells through epigenetic regulation of Sonic 
Hedgehog. Exp Cell Res. 2017;352:346-56.

7. Suzuki N, Yoshimura Y, Deyama Y, Suzuki K, Kitagawa Y. Mechanical stress directly suppresses osteoclast differentiation in RAW264.7 cells. Int J Mol Med. 2008;21:291-6.

8. Kadow-Romacker A, Hoffmann JE, Duda G, Wildemann B, Schmidmaier G. Effect of mechanical stimulation on osteoblast- and osteoclast-like cells in vitro. Cells Tissues Organs. 2009;190:61-8.

9. Wang J, Wang CD, Zhang N, Tong WX, Zhang YF, Shan SZ, Zhang XL, Li QF. Mechanical stimulation orchestrates the osteogenic differentiation of human bone marrow stromal cells by regulating HDAC1. Cell Death Dis. 2016;7:e2221.

10. Sun W, Zhao C, Li Y, Wang L, Nie G, Peng J, Wang A, Zhang P, Tian W, Li Q, Song J, Wang C, Xu X, Tian Y, Zhao D, Xu Z, Zhong G, Han B, Ling S, Chang YZ, Li Y. Osteoclast-derived microRNAcontaining exosomes selectively inhibit osteoblast activity. Cell Discov. 2016;2:16015.

11. Yuan FL, Wu QY, Miao ZN, Xu MH, Xu RS, Jiang DL, Ye JX, Chen FH, Zhao MD, Wang HJ, Li X. Osteoclast-Derived Extracellular Vesicles: Novel Regulators of Osteoclastogenesis and OsteoclastOsteoblasts Communication in Bone Remodeling. Front Physiol. 2018;9:628.

12. Park Y, Musson D, Naot D, Cornish J. Cell-cell communication in bone development and whole-body homeostasis and pharmacological avenues for bone disorders. Curr Opin Pharmacol. 2017;34:2135 .

13. Liu J, Li D, Wu X, Dang L, Lu A, Zhang G. Bone-derived exosomes. Curr Opin Pharmacol. 2017;34:649.

14. Yin P, Lv H, Li Y, Deng Y, Zhang L, Tang P. Exosome-Mediated Genetic Information Transfer, a Missing Piece of Osteoblast-Osteoclast Communication Puzzle. Front Endocrinol. 2017;8:336.

15. Kalluri R. VS LeBleu. (2020). The biology, function, and biomedical applications of exosomes. Science 367:eaau6977.

16. Liu M, Sun Y, Zhang Q. Emerging Role of Extracellular Vesicles in Bone Remodeling. J Dent Res. 2018;97:859-68.

17. Zhu S, Yao F, Qiu H, Zhang G, Xu H, Xu J. Coupling factors and exosomal packaging microRNAs involved in the regulation of bone remodelling. Biol Rev Camb Philos Soc. 2018;93:469-80.

18. Li Y, Jin D, Xie W, Wen L, Chen W, Xu J, Ding J, Ren D. Z Xiao. (2018). Mesenchymal Stem CellsDerived Exosomes: A Possible Therapeutic Strategy for Osteoporosis. Curr Stem Cell Res Ther 13:362-8.

19. Vitha AE, Kollefrath AW, Huang CC, Garcia-Godoy F. Characterization and Therapeutic Uses of Exosomes: A New Potential Tool in Orthopedics. Stem Cells Dev. 2019;28:141-50.

20. Deng LL, Wang YP, Peng Y, Wu Y, Ding YD, Jiang YH, Shen ZH, Fu Q. Osteoblast-derived microvesicles: A novel mechanism for communication between osteoblasts and osteoclasts. Bone. 2015;79:37-42.

21. Ota S, Zhou ZQ, Romero MP, Yang G, Hurlin PJ. (2016). HDAC6 deficiency or inhibition blocks FGFR3 accumulation and improves bone growth in a model of achondroplasia. Hum Mol Genet. 
22. Xiao F, Zhai Z, Jiang C, Liu X, Li H, Qu X, Ouyang Z, Fan Q, Tang T, Qin A, Gu D. Geraniin suppresses RANKL-induced osteoclastogenesis in vitro and ameliorates wear particle-induced osteolysis in mouse model. Exp Cell Res. 2015;330:91-101.

23. Chu D, Phuong T, Tien NB, Tran D, Thanh VV, Quang T, Truong D, Pham V, Ngoc V, Chu-Dinh T, Kushekhar K. An Update on the Progress of Isolation, Culture, Storage, and Clinical Application of Human Bone Marrow Mesenchymal Stem/Stromal Cells. Int J Mol Sci. 2020;21:e708.

24. Liu SY, Liu DW, Chen CD, Hamamura K, Moshaverinia A, Yang RL, Liu Y, Jin Y, Shi ST. MSC Transplantation Improves Osteopenia via Epigenetic Regulation of Notch Signaling in Lupus. Cell Metab. 2015;22:606-18.

25. Qin Y, Wang L, Gao Z, Chen G, Zhang C. Bone marrow stromal/stem cell-derived extracellular vesicles regulate osteoblast activity and differentiation in vitro and promote bone regeneration in vivo. Sci Rep. 2016;6:21961.

26. Najrana T, Mahadeo A, Abu-Eid R, Kreienberg E, Schulte V, Uzun A, Schorl C, Goldberg L, Quesenberry P, Sanchez-Esteban J. (2020). Mechanical stretch regulates the expression of specific miRNA in extracellular vesicles released from lung epithelial cells. J Cell Physiol.

27. Wang ZY, Maruyama K, Sakisaka Y, Suzuki S, Tada H, Suto M, Saito M, Yamada S, Nemoto E. Cyclic Stretch Force Induces Periodontal Ligament Cells to Secrete Exosomes That Suppress IL-1 $\beta$ Production Through the Inhibition of the NF-kB Signaling Pathway in Macrophages. Front immunol. 2019;10:1310.

28. Jiang C, Xiao F, Gu X, Zhai Z, Liu X, Wang W, Tang T, Wang Y, Zhu Z, Dai K, Qin A, Wang J. Inhibitory effects of ursolic acid on osteoclastogenesis and titanium particle-induced osteolysis are mediated primarily via suppression of NF-kappaB signaling. Biochimie. 2015;111:107-18.

29. Jimi E, Takakura N, Hiura F, Nakamura I, Hirata-Tsuchiya S. (2019). The Role of NF-kB in Physiological Bone Development and Inflammatory Bone Diseases: Is NF-кB Inhibition "Killing Two Birds with One Stone"? Cells 8.

30. Tan S, Wong J, Sim S, Tjio C, Wong K, Chew J, Hui J, Toh W. Mesenchymal stem cell exosomes in bone regenerative strategies-a systematic review. Mater Today Bio. 2020;7:eCollection.

31. Zuo R, Liu M, Wang Y, Li J, Wang W, Wu J, Sun C, Li B, Wang Z, Lan W, Zhang C, Shi C. Y Zhou. (2019). BM-MSC-derived exosomes alleviate radiation-induced bone loss by restoring the function of recipient BM-MSCs and activating Wnt/ $\beta$-catenin signaling. Stem Cell Res Ther 10:30.

32. Yun B, Maburutse B, Kang M, Park M, Park D, Kim Y, Oh S. Short communication: Dietary bovine milkderived exosomes improve bone health in an osteoporosis-induced mouse model. J Dairy Sci. 2020;103:7752-60.

33. Liu W, Li L, Rong Y, Qian D, Chen J, Zhou Z, Luo Y, Jiang D, Cheng L, Zhao S, Kong F, Wang J, Zhou Z, Xu T, Gong F, Huang Y, Gu C, Zhao X, Bai J, Wang F, Zhao W, Zhang L, Li X, Yin G, Fan J, Cai W. Hypoxic mesenchymal stem cell-derived exosomes promote bone fracture healing by the transfer of miR-126. Acta Biomater. 2020;103:196-212. 
34. Yang B, Kuang M, Kang J, Zhao J, Ma J, Ma X. Human umbilical cord mesenchymal stem cellderived exosomes act via the miR-1263/Mob1/Hippo signaling pathway to prevent apoptosis in disuse osteoporosis. Biochem Biophys Res Commun. 2020;524:883-9.

35. Grimm D, Grosse J, Wehland M, Mann V, Reseland JE, Sundaresan A. TJ Corydon. (2016). The impact of microgravity on bone in humans. Bone 87:44-56.

36. Li X, Han L, Nookaew I, Mannen E, Silva M, Almeida M, Xiong J. (2019). Stimulation of Piezo1 by mechanical signals promotes bone anabolism. eLife 8 .

\section{Figures}
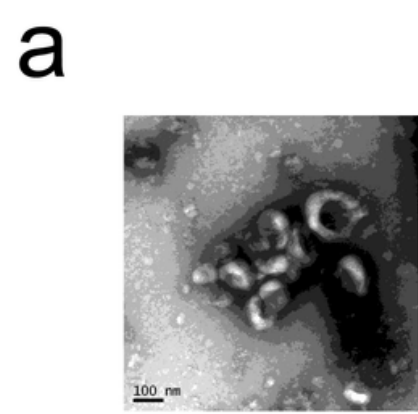

Static_Exos

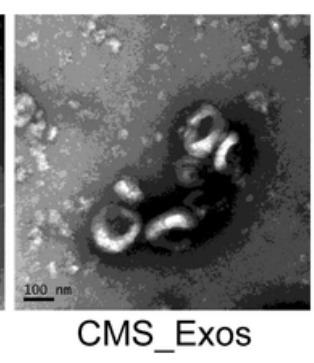

b

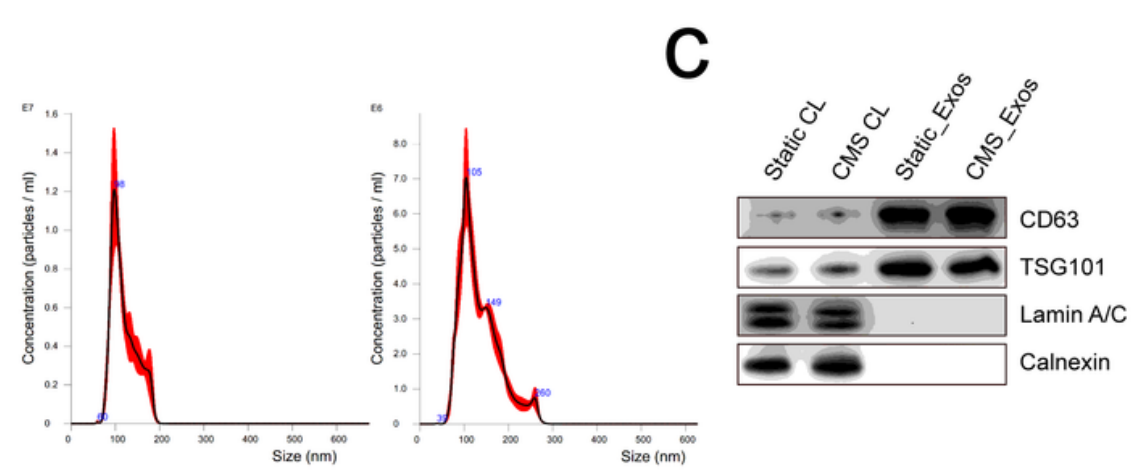

d

Hoechst 33342

DiO-labeled exosomes

Bright field
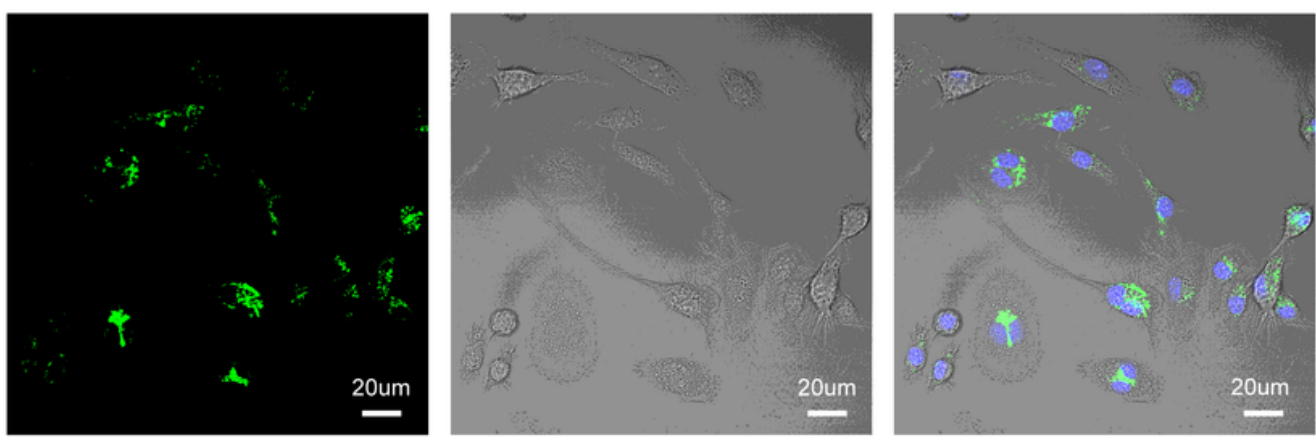

Static
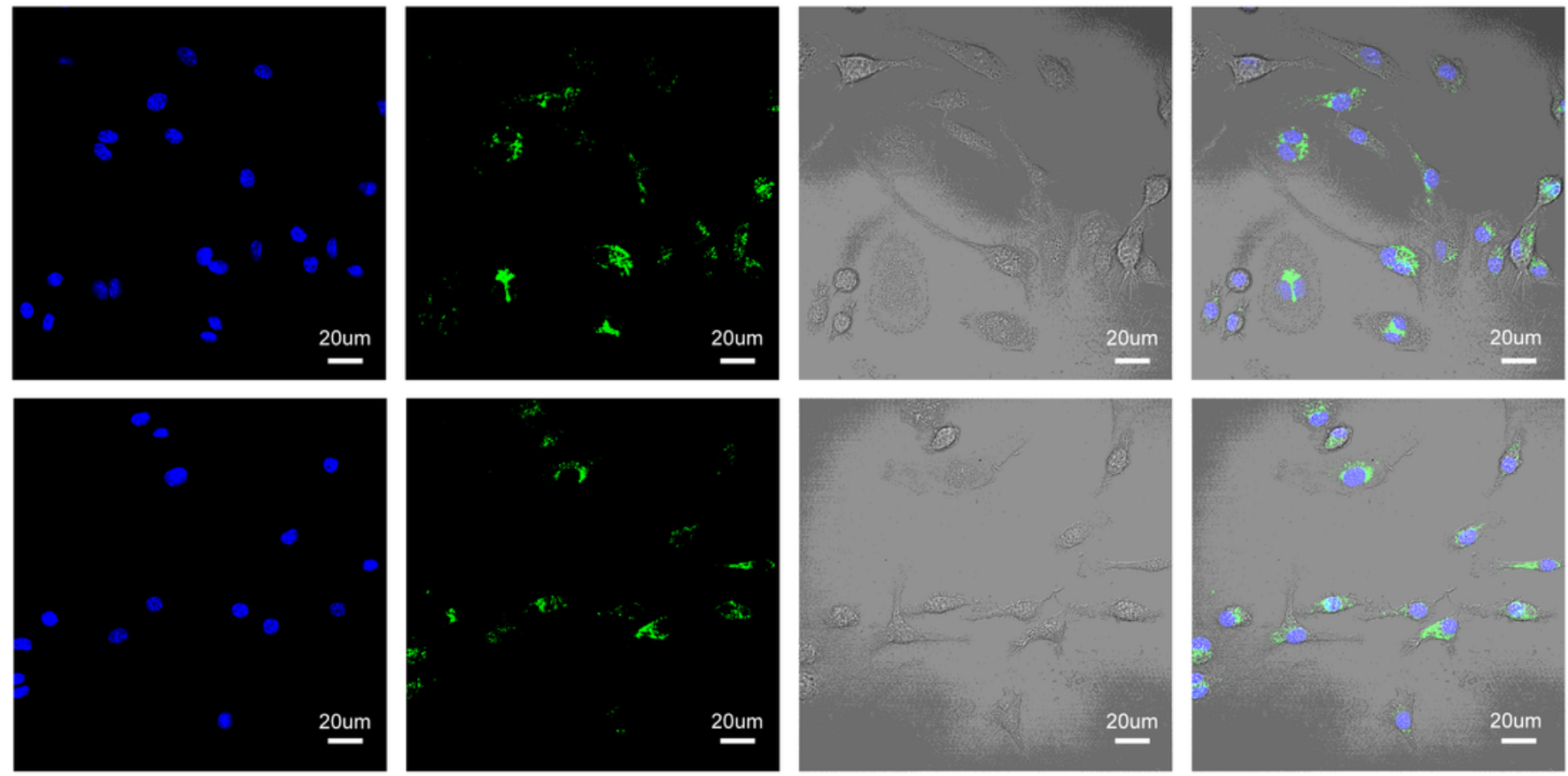

\section{Figure 1}

Characterization of exosomes and incorporation of exosomes into BMMs. (a) Electron microscopy images of exosomes. Scale bar, $100 \mathrm{~nm}$. (b) The size distribution and concentration of exosomes 
secreted by BMSCs measured by nanoparticle tracking analysis. (c) Western blotting for the exosomal markers CD63 and TSG101. (d) Confocal microscopy images showing the colocalization of exosomes from BMSCs with BMMs. Exosomes were labeled with 3,3'-dioctadecyloxacarbocyanine perchlorate (DiO) (green), and BMM cell nuclei were stained with Hoechst 33342 (blue).
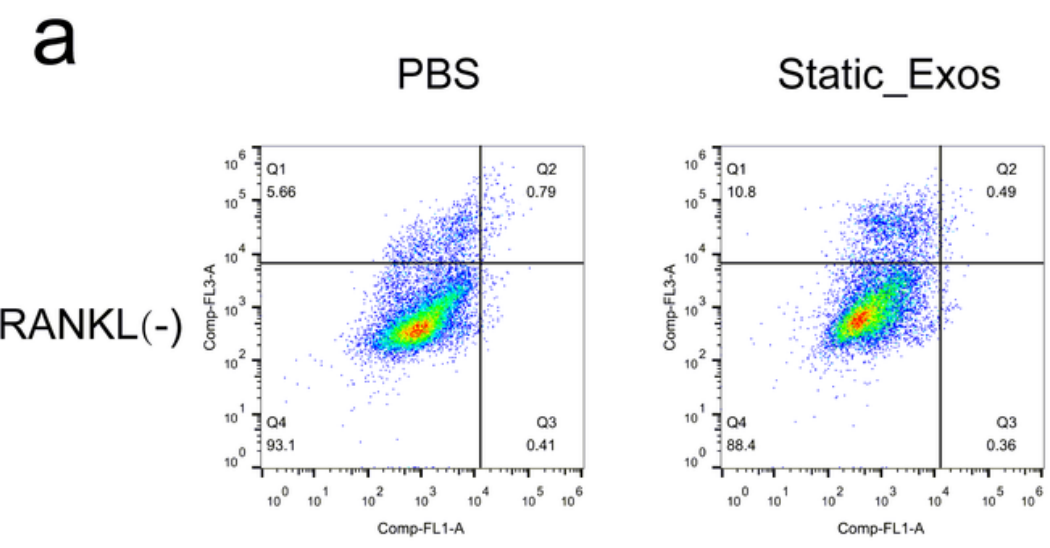

\section{CMS_Exos}

b
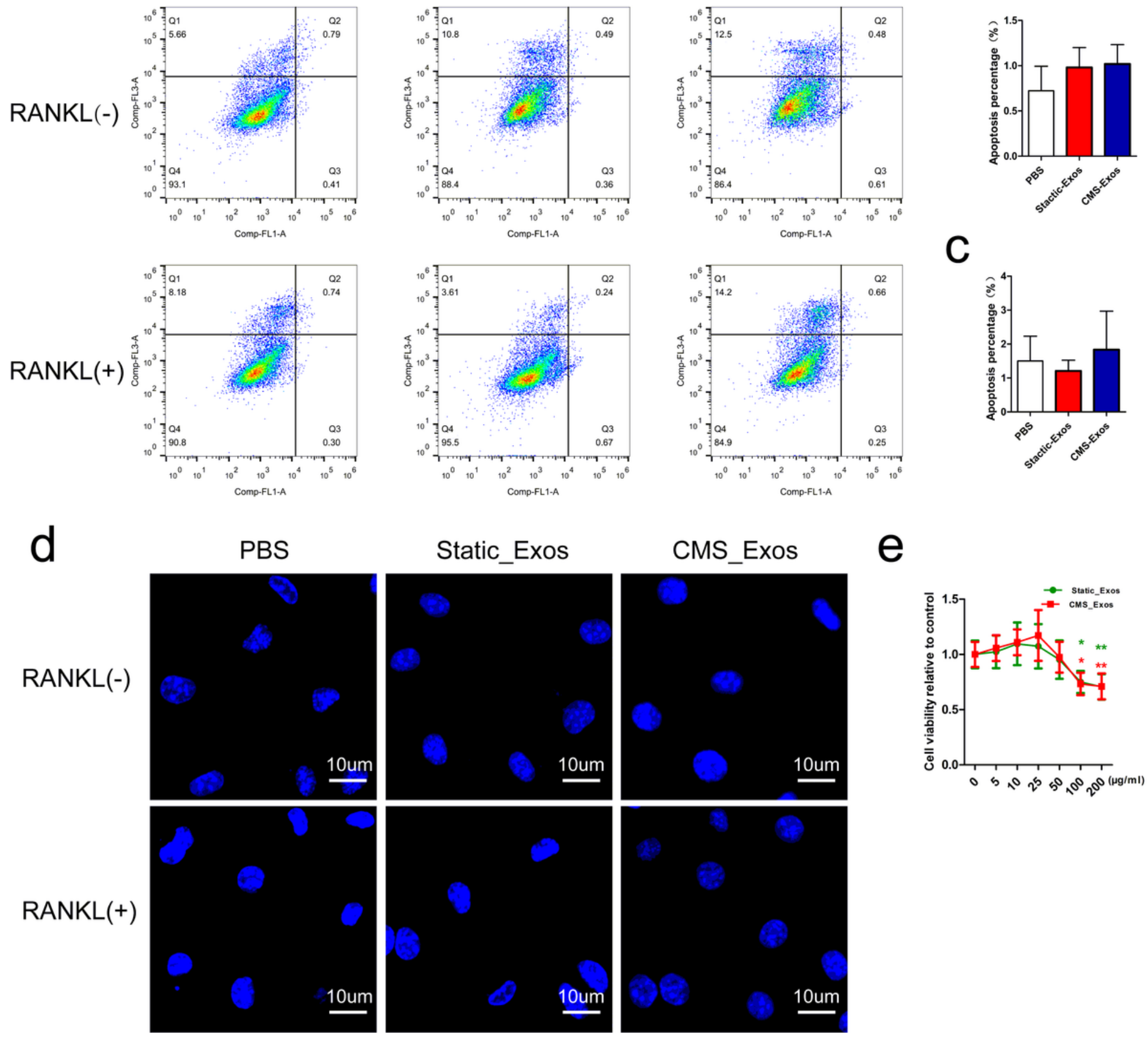

Static_Exos
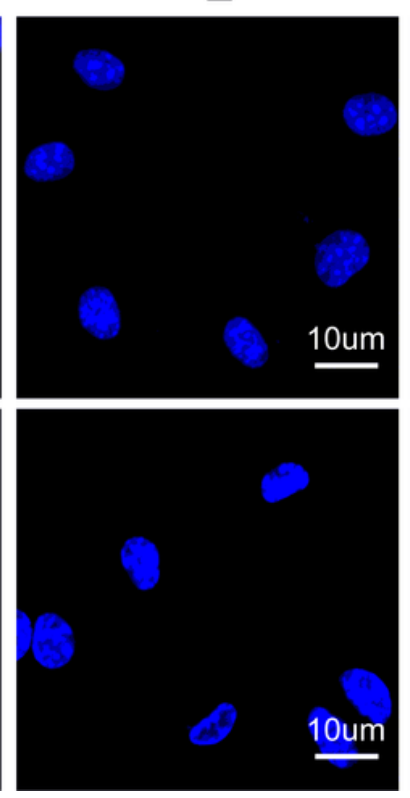

CMS_Exos
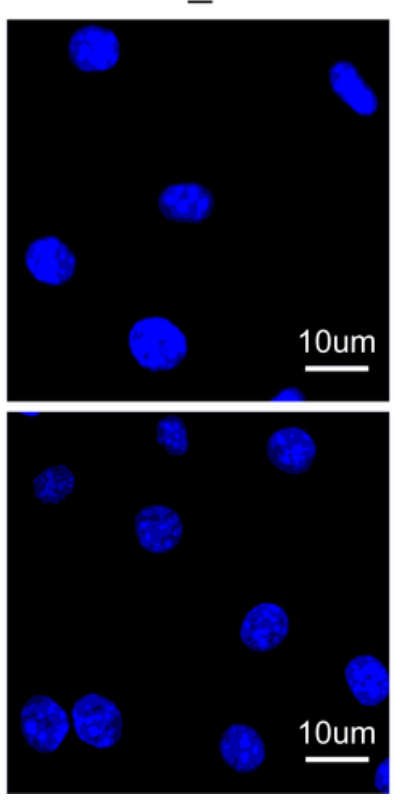

e

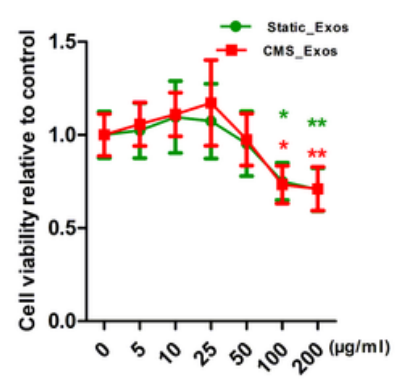

\section{Figure 2}

Exosomes do not affect BMM cell viability or induce cell apoptosis at low concentrations. (a) Flow cytometry analysis of the apoptosis rate of BMMs treated with $25 \mu \mathrm{g} / \mathrm{mL}$ exosomes for $48 \mathrm{~h}$. Quantitative analysis of the apoptosis rates of BMMs (b) not stimulated with RANKL or (c) stimulated with RANKL. (d) 
Exosomes did not induce nuclear fragmentation. BMMs were treated with the indicated exosomes for 48 $\mathrm{h}$ before the cells were fixed, stained with $0.1 \mu \mathrm{g} / \mathrm{mL}$ DAPI, and examined by fluorescence microscopy. (e) CCK-8 assay performed to analyze the cell viability of BMMs incubated with the indicated exosomes for $48 \mathrm{~h}$. All experiments were performed at least three times, and representative images are shown. Data are expressed as the mean $\pm S D ; * P<0.05$, ${ }^{*} P<0.01$.
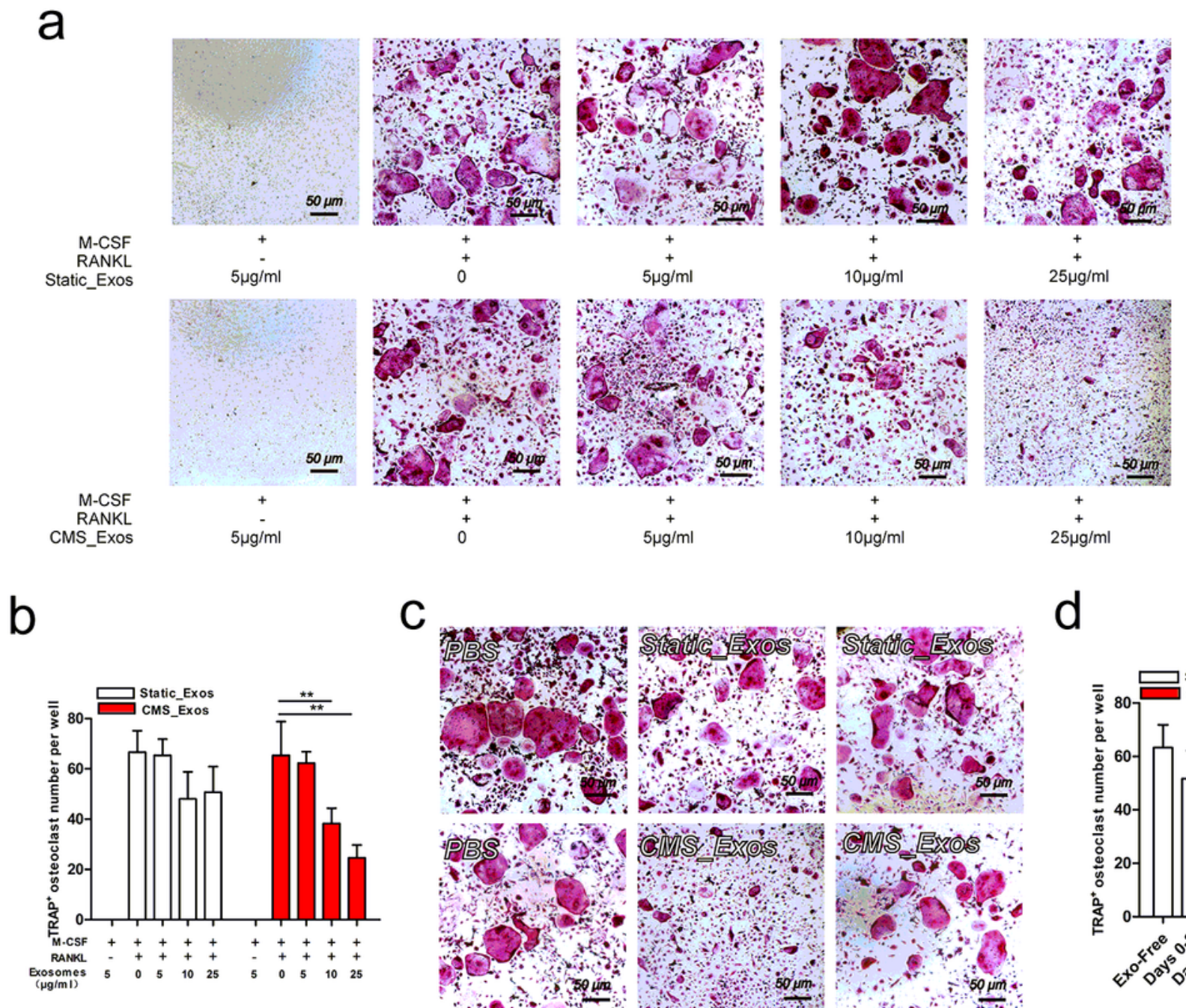

C

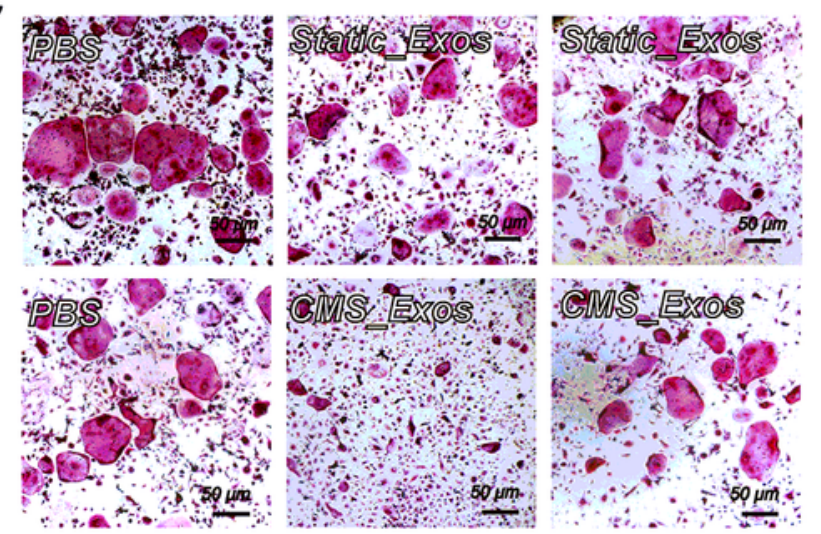

d

Exosome-Free

Days $0-2$

Days 3-5

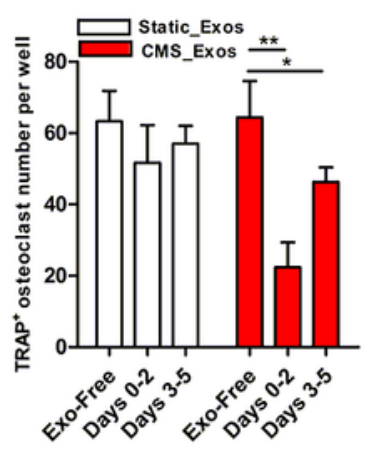

\section{e}

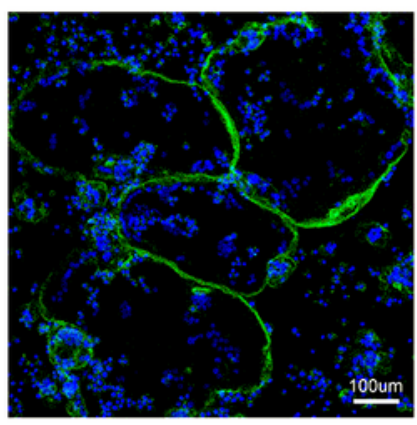

PBS

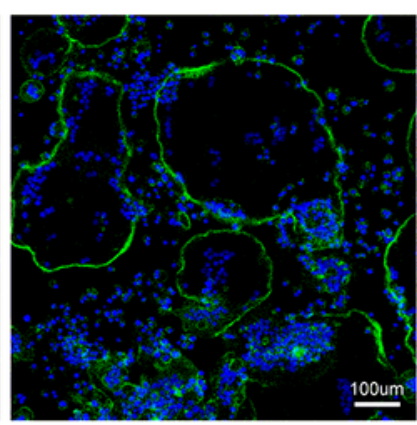

Static_Exos

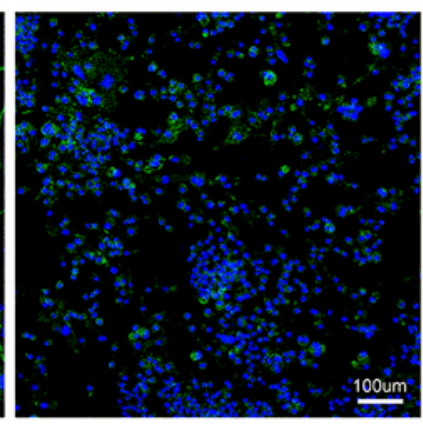

CMS Exos

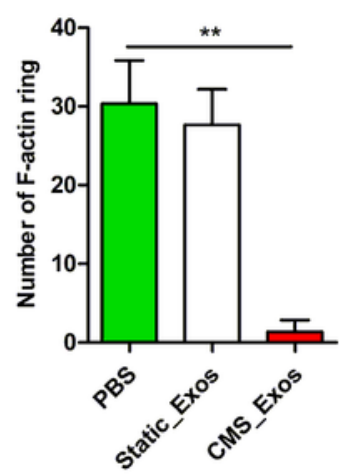

Figure 3 
CMS_Exos inhibit RANKL-induced osteoclastogenesis and actin ring formation. (a) Representative images of RANKL-stimulated BMMs stained for TRAP (red) after treatment with the indicated exosomes at different concentrations $(0,5,10$, or $25 \mu \mathrm{g} / \mathrm{mL})$. (b) The number of TRAP-positive multinucleated osteoclasts ( $\geq 3$ nuclei) was quantified. (c) Effect of the time of indicated exosome addition on osteoclast formation. BMM cells were stimulated with RANKL $(50 \mathrm{ng} / \mathrm{mL})$ alone or cotreated with the indicated exosomes $(25 \mu \mathrm{g} / \mathrm{mL})$ at different stages during a 5-day osteoclast culture. The cells were fixed and stained for TRAP activity. (d) The number of TRAP-positive multinucleated osteoclasts ( $\geq 3$ nuclei) was quantified. (e) BMMs were stimulated with RANKL and the indicated exosomes until mature osteoclasts formed. Then, the cells were fixed and stained for F-actin. The actin ring distribution was visualized by confocal microscopy. (f) The number of osteoclasts with an intact actin ring was counted. All experiments were performed at least three times, and representative images are shown. Data are expressed as the mean $\pm S D ; * P<0.05$, ** $P<0.01$.

a

NF-kB/p65

DAPI

Merged

RNAKL

Exosomes
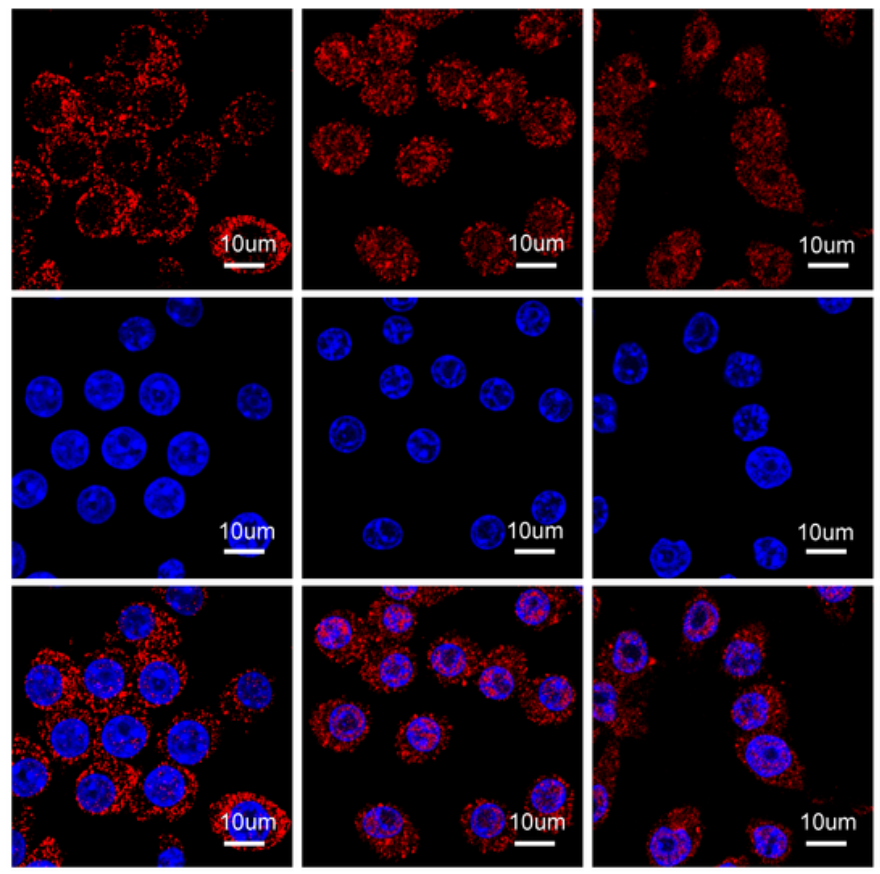

10um

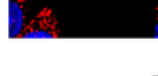

0

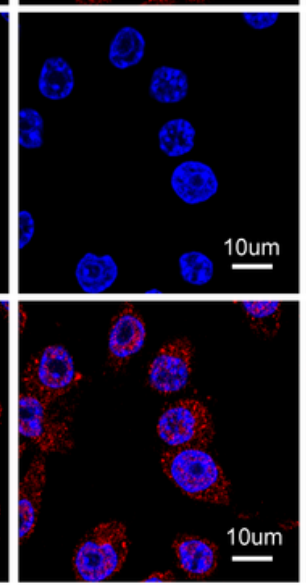

$+$

Static_Exos
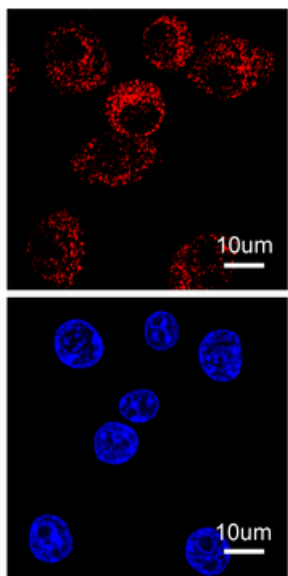

10um

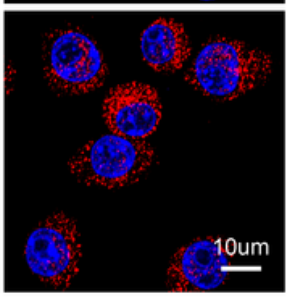

$+$

CMS_Exos b

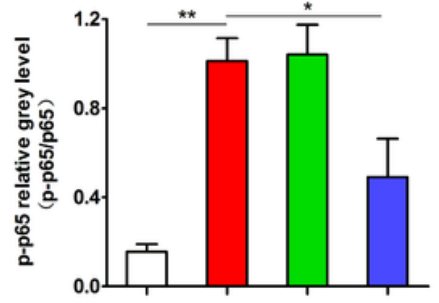

p-p65

p65

RANKL

C
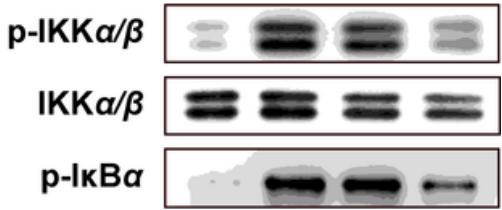

IкBa

GAPDH

RANKL

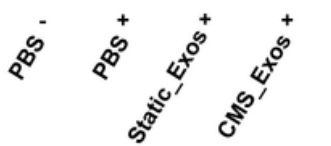

\section{Figure 4}

CMS_Exos inhibit the RANKL-induced activation of the NF-KB signaling pathway. (a) RAW264.7 cells were treated with the indicated exosomes for $4 \mathrm{~h}$, followed by stimulation with RANKL (50 ng/mL) for $20 \mathrm{~min}$. The localization of p65 was visualized by immunofluorescence staining. (b) and (c) Western blot analysis of the levels of the indicated proteins in BMMs pretreated with or without exosomes for $4 \mathrm{~h}$ prior to RANKL stimulation (50 ng/ml). The bar graph in (b) shows the relative expression of p-p65 calculated 
from the band intensity using ImageJ software. All experiments were performed at least three times, and representative images are shown. Data are expressed as the mean $\pm S D ; * P<0.05, * * P<0.01$.

a

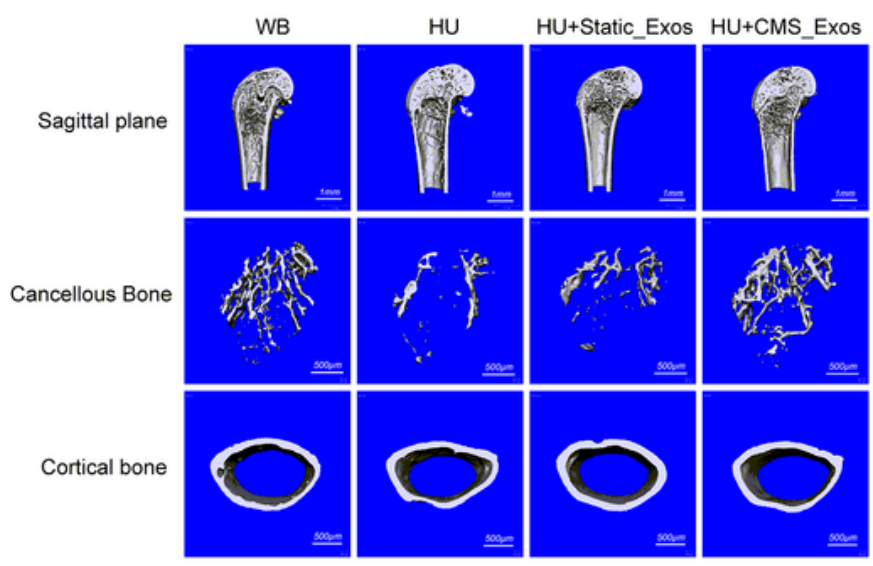

b

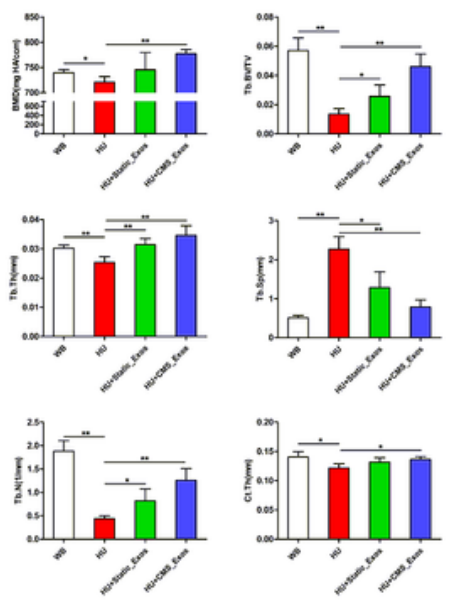

C

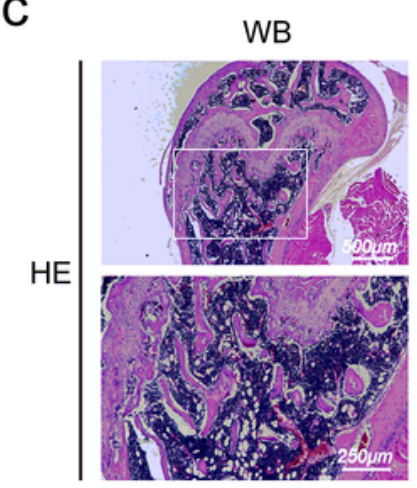

$\mathrm{HU}$

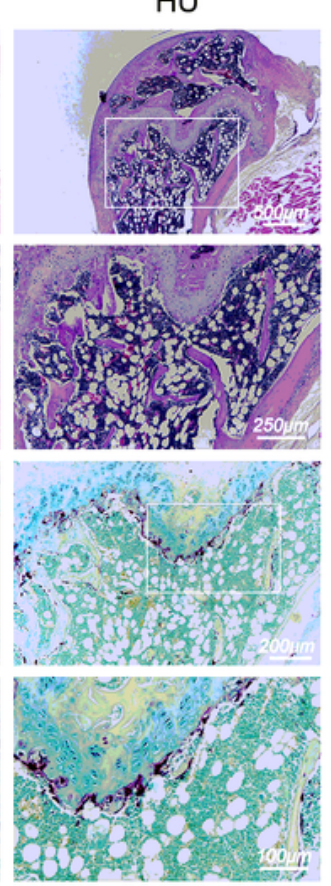

HU+Static_Exos

HU+CMS_Exos
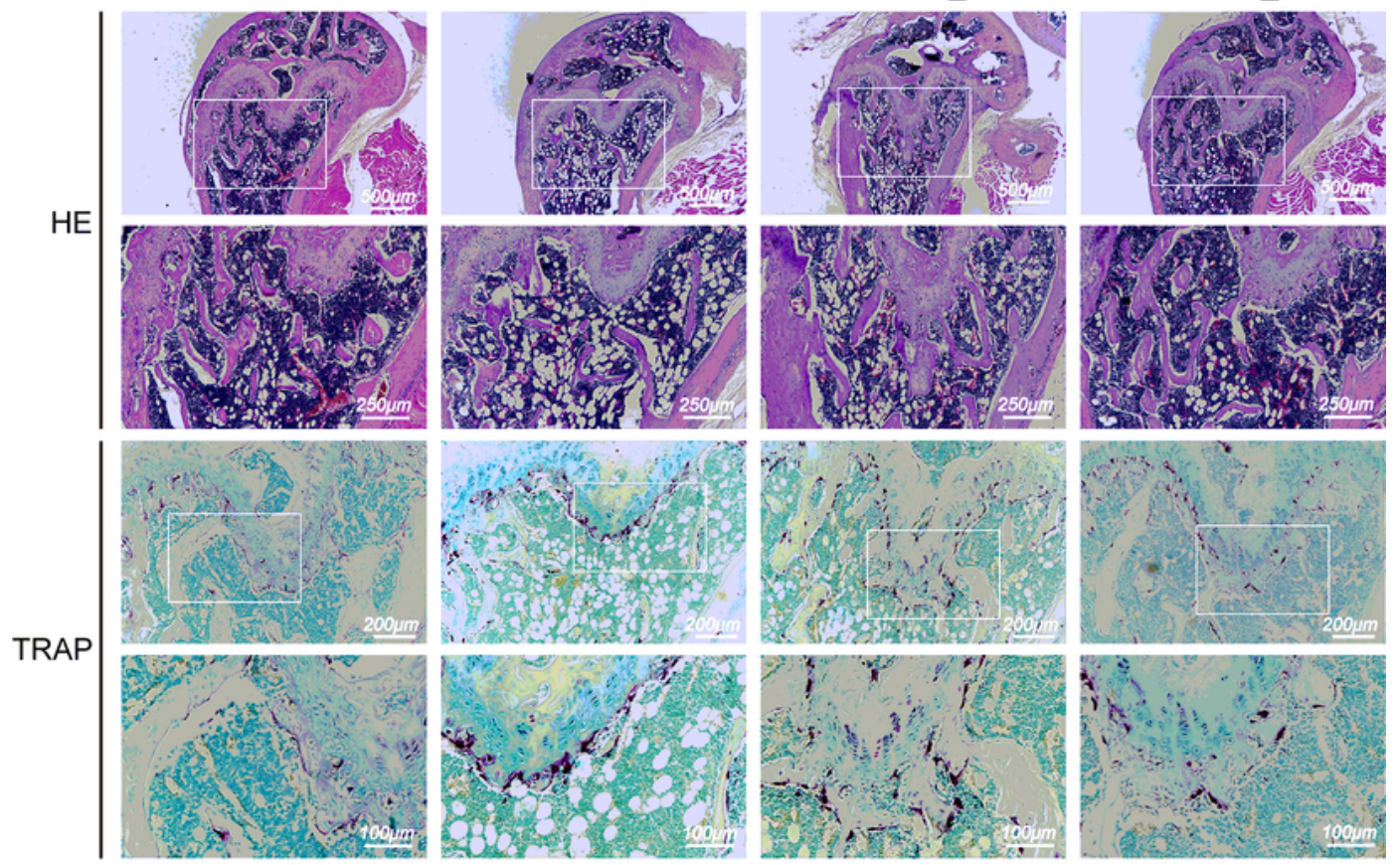

d
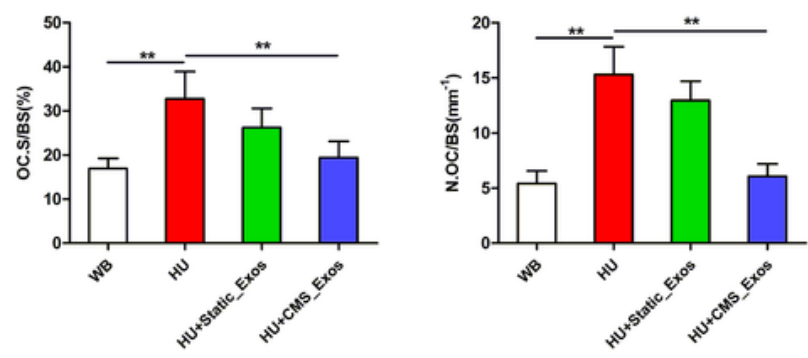

Figure 5

CMS_Exos prevented HU-induced bone loss. (a) Representative reconstructed micro-CT images of the distal femur in the indicated group. $n=5$. (b) Three-dimensional microstructural parameters of the distal femur in the indicated group. (c) Representative hematoxylin-eosin and TRAP staining images of the 
distal femur showing the bone volume in the indicated group. $n=5$. (d) The percentage of osteoclast surface per bone surface (OcS/BS, \%) and number of osteoclasts per field of tissue (No. of OCs per field) at $200 \times$ magnification were analyzed. Data are expressed as the mean $\pm S D ;{ }^{*} P<0.05,{ }^{*} P<0.01$. 